\title{
The intestinal contortin structure in Haemonchus contortus: An immobilised anticoagulant?
}

\author{
Peter Geldhof ${ }^{a, b}$, David Knox ${ }^{a, *}$ \\ ${ }^{a}$ Moredun Research Institute, Pentlands Science Park, Bush Loan, Penicuik, Midlothian, EH26 OPZ, UK \\ ${ }^{\mathrm{b}}$ Laboratory of Parasitology, Faculty of Veterinary Medicine, Ghent University, Salisburylaan 133, 9820 Merelbeke, Belgium
}

Received 17 March 2008; received in revised form 30 April 2008; accepted 2 May 2008

\begin{abstract}
Contortin was the first intestinal antigen of the sheep parasite Haemonchus contortus which induced significant levels of protection when used to vaccinate lambs. This antigen is present in the intestine of L4 and adult worms as a helical polymeric structure attached to the luminal surface of the intestinal cells. However, the nature of the protein itself and its function have never been reported. In the present study, contortin was isolated and analysed by peptide mass fingerprint and LC/MS-MS. These analyses indicated that contortin comprises two major proteins, Hc-PCP1 and Hc-PCP2, with homology to prolyl-carboxypeptidases. The two proteins show $64 \%$ amino acid sequence identity to each other and both are comprised of two prolyl-carboxypeptidase S28 type domains organised in a tandem repeat. The transcripts of both genes are present from the L4 stage onwards, coinciding with the onset of blood-feeding. Addition of contortin to a fibrinogen solution significantly inhibited blood coagulation in a dose-dependent manner. Mass-spectrometry indicated that the contortin-enriched fraction degraded the C-terminal end of the fibrinogen alpha-chain, which was shown previously to be essential for clot formation. The process happens within seconds after addition and can be inhibited by the dipeptidyl-peptidase IV inhibitors Diprotin A and Bt-PEG-Glu-Pro ${ }^{\mathrm{P}}(\mathrm{OPh})_{2}$. These data suggest that the prolyl-carboxypeptidases are intestinal anticoagulants used by $H$. contortus to interfere with blood coagulation.
\end{abstract}

(C) 2008 Australian Society for Parasitology Inc. Published by Elsevier Ltd. All rights reserved.

Keywords: Haemonchus contortus; Parasitic nematode; Blood-feeding; Anticoagulant; Prolyl-carboxypeptidase; Contortin

\section{Introduction}

Targeting proteins expressed on the gut surface has been a particularly successful approach for vaccine development in blood-feeding nematodes (Knox et al., 2003) and ectoparasites such as ticks (Willadsen et al., 1995). Munn (1977) first described contortin, using electron microscopy, as a helical polymeric extracellular structure present in large amounts in the intestine and the pharynx from the L4 and adult stage of the sheep parasite Haemonchus contortus. The polymer is associated with the luminal surface of the plasma membrane of the intestinal epithelium and its helical filaments fill the spaces between the microvilli. It is insoluble and can be enriched from

\footnotetext{
${ }^{*}$ Corresponding author. Tel.: + 44131 4455111; fax: +44 1314456111.

E-mail address: david.knox@moredun.ac.uk (D. Knox).
}

PBS extracts of adult parasites by ultracentrifugation (Munn, 1977). The function of contortin remains undefined, but Munn (1977) suggested that it could be an immobilised anticoagulant. When a contortin-enriched preparation (CEP) was used to vaccinate lambs, worm burdens were reduced by $78 \%$ following challenge infection (Munn et al., 1987). However, it was shown subsequently that CEPs contained a $110 \mathrm{kDa}$ major antigenic contaminant as judged by Western blotting despite only faint staining being evident in protein gels (Smith and Munn, 1990). This protein was purified using lectin-affinity chromatography and was subsequently defined as H11, the most effective immunogen isolated from a parasitic nematode to date (Knox et al., 2003). Subsequent effort focused on H11 and other gut proteins in Haemonchus and the exact nature and function of contortin was not investigated further. 
The aim of this study was to characterise contortin at the molecular level in an effort to elucidate its potential function.

\section{Materials and methods}

\subsection{Preparation of a contortin-enriched fraction}

CEP was prepared as previously described by Munn (1977). In short, adult $H$. contortus were homogenised in PBS $(1: 10, \mathrm{w} / \mathrm{v})$ and subsequently centrifuged for $5 \mathrm{~min}$ at $1000 \mathrm{~g}$. The supernatant was collected and the pellet re-homogenised in PBS followed by centrifugation at $1000 \mathrm{~g}$. The supernatants were pooled and centrifuged at $3000 \mathrm{~g}$ for $10 \mathrm{~min}$. The pellet was washed once with PBS and the pooled supernatants were centrifuged for $90 \mathrm{~min}$ at $10,000 \mathrm{~g}$. The pellets, which contain contortin, were resuspended in $1 \mathrm{ml}$ of PBS and stored at $-80^{\circ} \mathrm{C}$ prior to analysis.

\subsection{Gel electrophoresis and mass-spectrometry analysis}

An aliquot $(20 \mu \mathrm{l})$ of CEP was fractionated in a $10 \%$ SDS-PAGE gel under reducing conditions. The protein components were visualised by Coomassie Blue staining. All visible protein bands were excised from the gel and used for mass-spectrometry analysis. Protein bands were digested in-gel using trypsin, the resultant peptides subsequently isolated by HPLC using a reversed phase C18 PepMap column and finally analysed by MALDI-TOF massspectrometry and LC/MS-MS analysis. The Mascot search engine was used to statistically analyse the mass-spectrometry and LC/MS-MS data and to identify the proteins.

\subsection{Isolation and cloning of $c D N A s$}

The LC/MS-MS analyses identified the prominent component of CEP as prolyl-carboxypeptidases encoded by two expressed sequence tag (EST) clusters, HCC00232 and HCC00298 (www.nema.cap.ed.ac.uk/nematodeESTs/ nembase.html). Full length cDNA sequences of the prolyl-carboxypeptidases (PCPs; from hereon named $\mathrm{Hc}$ pcpl and Hc-pcp2) were isolated from a cDNA library made from 11-day-old $H$. contortus worms. Based on the consensus sequences of EST clusters, specific primers were designed to isolate the $5^{\prime}$ and $3^{\prime}$ ends of each cDNA. The primer sequences are shown in Table 1. The gene-specific primers were used in combination with the T3 cDNA library vector primer to amplify both full-length cDNA sequences. PCR products were cloned in pGEM-T (Promega) and sequenced. Sequence analyses and alignments were performed using DNAstar software (DNAstar Inc.).

\subsection{Bioinformatics}

The EST datasets of $H$. contortus and other nematodes were analysed using the Partigene bioinformatics pipeline (Parkinson et al., 2004) which is available on the NEMBASE
Table 1

PCR primer sequences used for reverse transcriptase-PCR experiments

\begin{tabular}{ll}
\hline $\begin{array}{l}\text { Haemonchus contortus } \\
\text { EST cluster (gene name) }\end{array}$ & Primer sequence \\
\hline HCC00232 (Hc-pcp1) & F RT-PCR $5^{\prime}$ aag cag gtt cag cct tt ca \\
& R RT-PCR $5^{\prime}$ ctt gcc cat tgt tcg tt tt \\
& $5^{\prime}$ RACE $5^{\prime}$ cat aat gac gca cga tcg ac \\
HCC00298 (Hc-pcp2) & F-RT-PCR 5 cgc tga cct gtg tca agt gt \\
& R RT-PCR $5^{\prime}$ aag tag ccg gct tcg tat tg \\
& $3^{\prime}$ RACE $5^{\prime}$ agc atc gcc gct ttc aat \\
\hline
\end{tabular}

website (www.nematodes.org/nematodeESTs/nembase. html). Further analyses of sequences and the deduced amino acid sequences were performed using DNAstar software (DNAstar Inc.). Database searches were performed on the NCBI server (www.ncbi.nlm.nih.gov/blast). Amino acid sequences were analysed for signal peptides and glycosylation sites using Signal P-(www.cbs.dtu.dk/services/ SignalP) and NetNGlyc-programs (www.cbs.dtu.dk/services/ NetNGlyc).

\subsection{Reverse transcriptase $P C R$}

Reverse transcriptase PCR (RT-PCR) was used to determine the stage-specific transcription of Hc-pcpl and Hc-pcp2. Total RNA was extracted from exsheathed L3, L4 and adult parasites using Total RNA Isolation Reagent (Advanced Biotechnologies Ltd.). The RT-PCRs were carried out using the Superscript One-Step RT-PCR System (Invitrogen). The specific primers used for detection of the Hc-pcp1 of the Hc-pcp2 transcripts are shown in Table 1. After 35 cycles of amplification, the RT-PCR products were separated on $1 \%$ agarose gels. A cytoplasmic superoxide dismutase gene (SODc; acc. Z69621; Liddell and Knox, 1998) was used to control for the efficiency of the RNA purifications.

\subsection{S28 peptidase activity assays}

Dipeptidyl peptidase (DPP) activity was measured using the chromogenic substrates H-Ala-Pro- $p$ Nitroanilide (DPP IV, 1.0 $\mathrm{M}$ in methanol; Bachem) and H-Lys-Ala- $p$ Nitroanilide (DPP II, 1.0 M in methanol; Bachem). Different quantities of CEP $(10-30 \mu \mathrm{g})$ were mixed with the substrates (final conc. $1 \mathrm{mM})$ in $100 \mu \mathrm{l}$ of buffer ranging from $\mathrm{pH} 3$ to $8(\mathrm{pH}$ 3-5: 0.1 M sodium acetate buffer, $\mathrm{pH}$ 6-7: 0.1 M phosphate buffer, $\mathrm{pH}$ 8: $0.1 \mathrm{M}$ Tris buffer). Samples were incubated for $45 \mathrm{~min}$ at $37^{\circ} \mathrm{C}$ when the optical density was measured at $405 \mathrm{~nm}$. The effect of DPP IV inhibitors, Diprotin A and Diprotin B (Bachem), on activity was determined by adding different concentrations of the individual inhibitors $(10,100$, 500 and $1 \mathrm{mM}$ ) to the reaction mixture described above. All experiments were performed in duplicate.

\subsection{Fibrin clotting/re-aggregation assay}

A bovine fibrin solution (concentration $15 \mathrm{mg} / \mathrm{ml}$ ) (Sigma) was clotted by the addition of $1 \mu \mathrm{l}$ of a thrombin 
stock solution $(10 \mathrm{mg} / \mathrm{ml})$ (Sigma) and incubated at $37^{\circ} \mathrm{C}$ for $15 \mathrm{~min}$. The fibrin clot was collected and resuspended in $8 \mathrm{ml}$ of $1 \mathrm{M} \mathrm{NaBr} / 0.05 \mathrm{Na}$ acetate buffer $\mathrm{pH}$ 5.3. The resultant fibrin monomer solution was subsequently concentrated to $750 \mu \mathrm{l}$ and stored at $4{ }^{\circ} \mathrm{C}$ prior to use.

Re-aggregation of the fibrin solution was carried out by mixing $5 \mu \mathrm{l}$ of the fibrin monomer solution with $95 \mu \mathrm{l}$ of PBS. The re-aggregation was monitored in a fluorimeter by measuring the scattered light at a $90^{\circ}$ angle at $350 \mathrm{~nm}$. Results are shown as the change in scattered light over a $10 \mathrm{~min}$ period. The effect of CEP on the fibrin re-aggregation was assessed by incubating $5 \mu$ of the fibrin monomer solution with different quantities of CEP for $30 \mathrm{~min}$ at $37{ }^{\circ} \mathrm{C}$ prior to the fluorimetric analysis. The effect of the DPP IV inhibitor Diprotin A was determined by adding $0.4 \mu \mathrm{l}$ of a $0.5 \mathrm{M}$ inhibitor stock solution to the reaction mixture described above. All incubations were done in the presence of the inhibitors (all from Sigma), Aminoethylbenzenesulfonyl fluoride hydrochloride (AEBSF, $1 \mathrm{mM})$, Pepstatin $(0.1 \mathrm{mM})$, trans-epoxysucciny-L-leucylamido (4-guanidino) butane (E64, $0.1 \mathrm{mM}$ ) and 1.10 Phenanthroline (1, $10 \mathrm{Phe}, 50 \mathrm{mM})$ to block the activity of serine, aspartyl, cysteine and metallo-proteases, respectively.

\subsection{Fibrin degradation assay}

Twenty-five micrograms of fibrin monomers, prepared as described above, were mixed with different amounts of CEP and incubated at $37^{\circ} \mathrm{C}$ for different lengths of time, ranging from $15 \mathrm{~s}$ to $30 \mathrm{~min}$. The protease inhibitors AEBSF, Pepstatin, E64 and 1.10 Phe were included in the reaction mixtures at final concentrations described above. Samples were subsequently analysed on $10 \%$ SDS-PAGE gels under reducing conditions and visualised by Coomassie Blue staining. The effects of the DPP IV-specific inhibitors Diprotin A and Bt-PEG-Glu-Pro ${ }^{\mathrm{P}}(\mathrm{OPh})_{2}$ (Gilmore et al., 2006) were investigated by adding the inhibitors to the reaction mixture described above at a final concentration of $0.1 \mathrm{M}$ and $50 \mu \mathrm{M}$, respectively. The inhibitors were preincubated with contortin for $30 \mathrm{~min}$ prior to addition of fibrin monomers.

\subsection{Labelling of CEP with Bt-PEG-Glu-Pro ${ }^{P}(\mathrm{OPh})_{2}$}

Ten micrograms of CEP was mixed with $50 \mu \mathrm{M}$ of the DPP IV-specific biotinylated inhibitor Bt-PEG-Glu-Pro${ }^{\mathrm{P}}(\mathrm{OPh})_{2}$ (Gilmore et al., 2006) and incubated for $15 \mathrm{~min}$ at $37{ }^{\circ} \mathrm{C}$. Samples were subsequently run onto a $10 \%$ SDS-PAGE under reducing conditions and then blot transferred onto a polyvinylidene fluoride (PVDF) membrane. Membranes were blocked in $10 \%$ horse serum in PBS containing $0.05 \%$ Tween 20 (PBST) for $1 \mathrm{~h}$ and then incubated with conjugated streptavidin (Sigma) diluted 1:5000 in PBST for $1 \mathrm{~h}$. Bands were visualised by adding $0.05 \%$ 3,3 diaminobenzidine tetrachloride in PBS containing $0.01 \% \mathrm{H}_{2} \mathrm{O}_{2}$. CEP incubated without the addition of the inhibitor served as the negative control.

\section{Results}

\subsection{Characterisation of the contortin-enriched fraction (CEP)}

The protein profile of the CEP is shown in Fig. 1. The prominent band (arrowed in Fig. 1) and the remaining visible bands were excised from a Coomassie-stained gel, analysed by LC/MS-MS after a tryptic digest and then used to generate a peptide mass fingerprint which was screened against all the $H$. contortus EST datasets available on the Nembase website. The prominent band yielded three peptide sequences which showed a $100 \%$ match with clusters HCC00232 and HCC00298 (Table 2). Clusters HCC00232 and HCC00298, respectively, contain 421 and 37 individual EST sequences. The consensus sequence of cluster HCC00232 was 2579 bp long. An additional $884 \mathrm{bp}$ at the $5^{\prime}$ end was isolated from a cDNA library by a PCR approach using gene-specific primers in combination with the T3 vector primer. This resulted in a fulllength coding sequence which encodes a $122 \mathrm{kDa}$ protein.

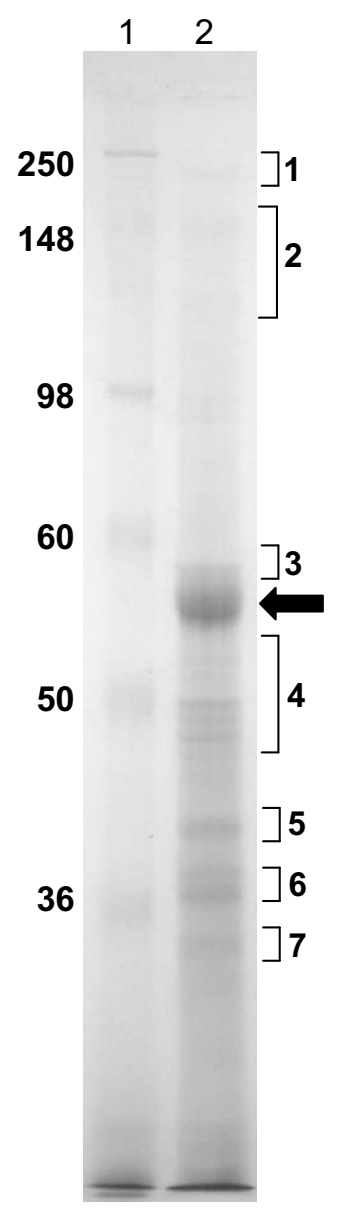

Fig. 1. Ten percent SDS-PAGE profile of the contortin-enriched protein fraction (CEP) under reducing conditions. Lane 1: marker, lane 2: CEP. The numbered zones and the prominent band around $55 \mathrm{kDa}$ (arrow) were excised and analysed by tryptic digest, peptide mass fingerprint analysis and LC-MS/MS analysis. 
Table 2

Peptide sequences from the LC-MS/MS analysis of the $55 \mathrm{kDa}$ band

Peptides EST cluster

LDYHEYYQVVEASIR DFDEEGWASVDR FDFWEGTQFAEDIYR DFDEEGWASVDR

Cluster HCC00298 has a consensus sequence of $3037 \mathrm{bp}$. Together with an additional $562 \mathrm{bp}$ at the $3^{\prime}$ end, which was isolated in a similar approach as described above, it codes for a $129 \mathrm{kDa}$ protein. Both proteins show homology to PCP-like proteins in Caenorhabditis elegans (PCP-2 acc. NP_501599 and PCP-3 acc. NP_501598.1). The shared identity between the $H$. contortus and $C$. elegans proteins was approximately $38 \%$. The two $H$. contortus proteins are herein named Hc-PCP1 (cluster HCC00232) and HcPCP2 (cluster HCC00298). An alignment of Hc-PCP1 and Hc-PCP2 is shown in Fig. 2A. The two proteins show $64 \%$ identity and $77 \%$ similarity to each other in amino acid sequence. Both contain a predicted signal peptide, albeit at a different location. Both proteins also contain multiple putative glycosylation sites, marked in Fig. 2A. Both Hc-PCP1 and Hc-PCP2 are comprised of two serine peptidase S28 type domains (pfam05577) organised in a tandem repeat. This feature seems to be nematode-specific; only the C. elegans PCPs show a similar structure. Each of the S28 domains encode a $51 \mathrm{kDa}$ peptidase, which approximately matches the size of the prominent band on SDS-PAGE gels (arrowed in Fig. 1). Therefore, it is likely that the two tandem structured PCPs are post-translationally processed into the four individual prolyl-carboxypeptidases, from hereon termed PCP1A, PCP1B, PCP2A and PCP2B. Interestingly, Hc-PCP2 has a unique C-terminal extension which is proline-rich and hydrophobic. The exact function of this part of the protein is unknown, but it might be involved in anchoring the protein to a cell membrane.

Fig. 2B shows a partial sequence alignment of the region around the active sites of the different $H$. contortus PCPs and human PCP. Catalysis by this type of peptidase is dependent on three active site residues, namely serine, histidine and aspartic acid which, although being dispersed throughout the primary protein sequence, come together in close proximity to form the active site when the enzyme is folded correctly. The catalytic residues in the Haemonchus PCP sequences here are ordered serine, aspartic acid and histidine and this feature, alongside the homology to PCPs place these enzymes in the clan SC, family S28 (Underwood et al., 1999). The region around the putative active site serine is highly conserved with $6 / 10$ residues identical in all the comparators, this number diminishing to $3 / 10$ and $1 / 8$ around the putative active site aspartic acid and histidine, respectively. Of the PCP domains, PCP 1A showed closest homology to PCP2B - 69.4\% identity with $81.8 \%$ similarity. Comparisons between other domain combinations all resulted in $\sim 30 \%$ identity, $50 \%$ similarity. All domains showed around 19\% identity and 30\% similarity to DPP IV of human origin and $25 \%$ identity, $40 \%$ similarity to the PCP of human origin.
Peptide mass fingerprint and LC/MS-MS analysis of additional protein bands (numbered in Fig. 1) resulted in the identification of a further eight proteins present in CEP (Table 3). These include a myosin, the aminopeptidase H11, glutamate dehydrogenase, apical gut membrane protein, metallo-peptidase 3, galectin and cysteine proteinases.

The full-length $H$. contortus PCP1 and PCP2 sequences were also used to search EST databases of other parasitic nematodes. EST clusters encoding PCPs were identified in Ancylostoma caninum (cluster ACC04826, two ESTs), Ascaris suum (cluster ASC18950, one EST), Onchocerca volvulus (cluster OVC01220, one EST), Strongyloides ratti (cluster SRC00324, six ESTs) and Parastrongyloides trichosuri (cluster PTC00674, one EST). Sequence homology varied between $32 \%$ and $42 \%$ over a maximum of 205 amino acids.

\subsection{Transcription profile of the Hc-pcp's}

Hc-pcp1 and Hc-pcp2-specific primers were used in RT-PCR on total RNA samples from exsheathed L3, L4, 11- and 22-day-old parasites (Fig. 3). Transcripts of the two genes were present from the L4 stage onwards whilst SODc was transcribed by all the parasite stages.

\subsection{8 peptidase activity assays}

At $\mathrm{pH} 7.5$, CEP hydrolysed the DPP IV-specific substrate in a dose-dependent manner (Fig. 4A). The optimal activity was at $\mathrm{pH} 6$ (Fig. 4B) and this was partially inhibited by Diprotin A (H-Ile-Pro-Leu; Fig. 4C) in a dose-dependent manner but unaffected by Diprotin B. (H-Val-Pro-Leu-OH). No activity could be detected against the DPP II substrate (results not shown).

\subsection{Coagulation assays}

Mentlein and Heymann (1982) reported previously that DPP IV inhibited the polymerisation of fibrin monomers. Therefore, a coagulation assay with purified fibrinogen was used to analyse if CEP showed anti-clotting activity and if it was targeted against fibrinogen. The results of this fibrin re-aggregation assay are shown in Fig. 5A. The addition of CEP to a fibrin monomer solution significantly inhibited the re-aggregation in a dose-dependent manner, a $55 \%$ and $75 \%$ reduction with 2.5 and $5 \mu 1$, respectively, as measured by the change in scattered light over a $10 \mathrm{~min}$ period. This effect was substantially overcome by the addition of the DPP IV inhibitor Diprotin A (Fig. 5B). Addition of specific inhibitors for all four classes of proteinases (serine, cysteine, metallo and aspartyl proteases) did not inhibit the anti-clotting activity. 


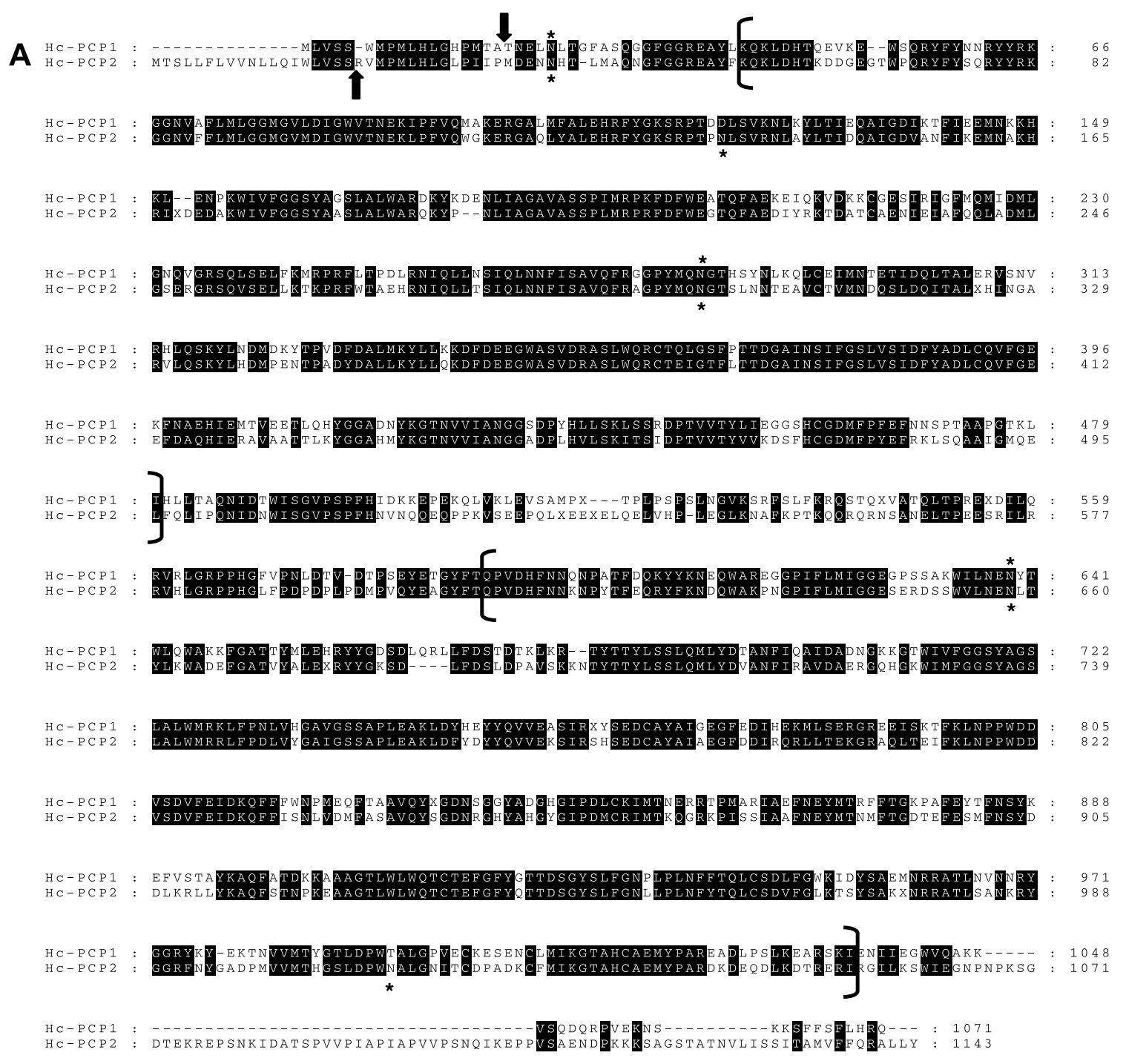

B

\begin{tabular}{|c|c|c|}
\hline CP1A & VFGGSYAGSLALWARDKYKDENLIAGAVASSPIMRPKFDFWEA---TQFAEKE] & \\
\hline PCP2A & AKWIVFGGSYAASLALWARQKYP--NLIAGAVASSPLMRPRFDFWEG---TQFAEDIYRK & 172 \\
\hline PCP1B & GTWIVFGGSYAGSLALWMRKLFP--NLVHGAVGSSAPLEAKLDYHEY---YQVVEASIRX & 175 \\
\hline PCP2B & GKWIMFGGSYAGSLALWMRRLFP--DLVYGAIGSSAPLEAKLDFYDY---YQVVEKSIRS & 173 \\
\hline \multirow[t]{2}{*}{ Human } & QPVIAIGGSYGGMLAAWFRMKYP--HMVVGALAASAPIWQFEDLVPCGVFMKIVTTDFRK & 8 \\
\hline & $\therefore: *^{*}::^{*} \cdot:$ & \\
\hline PCP1A & ADLCQVFG---EKFNAEHIEMTVEETLQHYGGADNY-KGTNVVIANGGSDPYHLLSKLSS & 4 \\
\hline PCP2A & ADLCQVFG---EEFDAQHIERAVAATTLKYGGAHMY-KGTNVVIANGGADPLHVLSKITS & 405 \\
\hline PCP1B & TQLCSDLFGWKIDYSAEMNRRATLNVNNRYGGRYKY-EKTNVVMTYGTLDPWTALGPVEC & 411 \\
\hline PCP2B & TQLCSDVFGLKTSYSAKBNRRATLSANKRYGGRFNYGADPMVVMTHGSLDPWNALGNITC & 410 \\
\hline \multirow[t]{2}{*}{ Human } & SDDC-------FQQWGVRPRPSWITTMYGGKNIS-SHTNIVFSNGELDPWSGGGVTKD & 440 \\
\hline & . $: *:: \star \star \star$ & \\
\hline PCP1A & 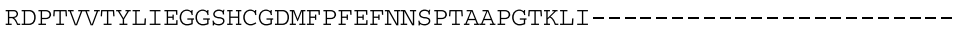 & \\
\hline PCP2A & 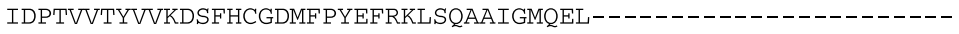 & \\
\hline PCP1B & K-ESENCLMIKGTAHCAEMYPAREADLPSLKEARSKIENIIEGWVQAKKVSQDQRPVEKN & $\mathrm{I}$ \\
\hline PCP2B & 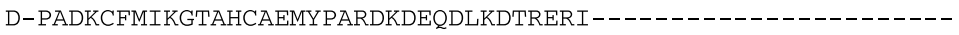 & 44 \\
\hline Human & ITDTLVAVTISEGAHHLDLRTKNALDPMSVLLARSLEVRHMKNWIRDFYDSAGKQH---- & 96 \\
\hline
\end{tabular}

Fig. 2. The figure shows a pairwise alignment of the derived amino acid sequences from the two Haemonchus prolyl-carboxypeptidase (PCP)-encoding transcripts (A) and a comparison of the active site regions of the individual PCP domains they encode (B). Predicted cleavage sites of the N-terminal signal sequences in both proteins are marked with an arrow. Putative glycosylation sites are marked with an * (A). Both proteins contain two prolylcarboxypeptidase S28 type domains which are marked with []. A partial sequence alignment of the region around the active sites of the different H. contortus PCPs and human PCP is shown in (B). The residues around the active site serine are shown in bold, the residues around the aspartic acid site by single underline and the histidine site in double underline. The order of these active site residues places the PCPs in the peptidase clan SC, family S28. 
Table 3

Additional proteins identified in the contortin-enriched protein fraction by LC/MS-MS

\begin{tabular}{ll}
\hline $\begin{array}{l}\text { Zone on SDS-PAGE } \\
\text { gel (Fig. 1) }\end{array}$ & Protein ID \\
\hline 1 & $\begin{array}{l}\text { Myosin (DQ310759) } \\
\text { Integral membrane protein H11 (X94187) } \\
\text { Glutamate dehydrogenase (Hc-gldh1, } \\
3\end{array}$ \\
& AF000967) \\
4 & Apical gut membrane protein (GA1, U55864) \\
& and metallo-peptidase (MEP III, AF080172) \\
5 & Cysteine proteinase (HaeCytPro2 - M80386) \\
6 & Galectin (Hco-gal-1, AF077944) \\
7 & Cysteine proteinase (GCP7, AF046229) \\
\hline
\end{tabular}

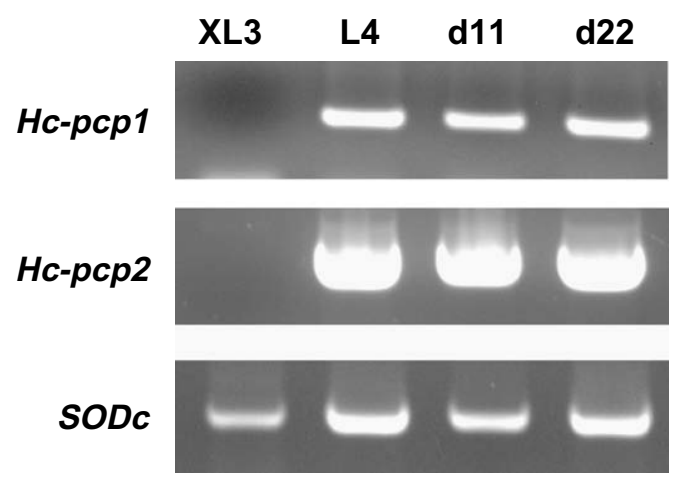

Fig. 3. Presence of $H c-p c p 1$ and $H c-p c p 2$ transcripts as revealed by reverse transcriptase (RT)-PCR. The lanes are independent RT-PCR reactions using target RNA from exsheathed L3, L4, 11- and 22-day-old adult parasites. RT-PCR for a cytoplasmic superoxide dismutase $(S O D c)$ was used as a control to check the uniformity of the RNA purifications.

\subsection{Fibrin degradation assay}

SDS-PAGE gel analysis of the fibrin monomer solution under reducing conditions (Fig. 6A lane 1) revealed three protein bands of approximately 63,56 and $47 \mathrm{kDa}$, the $\alpha$-, $\beta$ - and $\gamma$-chains, respectively. The addition of CEP resulted in the proteolytic degradation of both the $\alpha$ - and $\beta$-chain after $15 \mathrm{~s}$ of incubation at $37^{\circ} \mathrm{C}$ (lane 2). The addition of specific inhibitors for all four classes of proteinases (serine, cysteine, metallo and aspartyl proteases) stopped the degradation of the $\beta$-chain, but not the $\alpha$-chain (lane 3). However, preincubating CEP with the DPP IV-specific inhibitors Diprotin A (lane 4) and Bt-PEG-Glu-Pro${ }^{\mathrm{P}}(\mathrm{OPh})_{2}$ (lane 5) completely abolished the degradation of the fibrinogen $\alpha$-chain.

The specific degradation of the $\alpha$-chain coincided with an intensification of the $\gamma$-chain, as shown in lane 3 (arrow), suggesting the presence of proteolytic degradation products of the $\alpha$-chain. Mass-spectrometric fingerprint and LC-MS/MS analysis on this protein band revealed the presence of both the $\gamma$ - and $\alpha$-chain, whereas in the $\gamma$ band of an untreated sample no peptides of the $\alpha$-chain were present. Fig. 6B shows the location of the identified peptides in the complete $\alpha$-chain protein sequence. No peptides could be identified in the $\mathrm{C}$-terminal part of the pro-
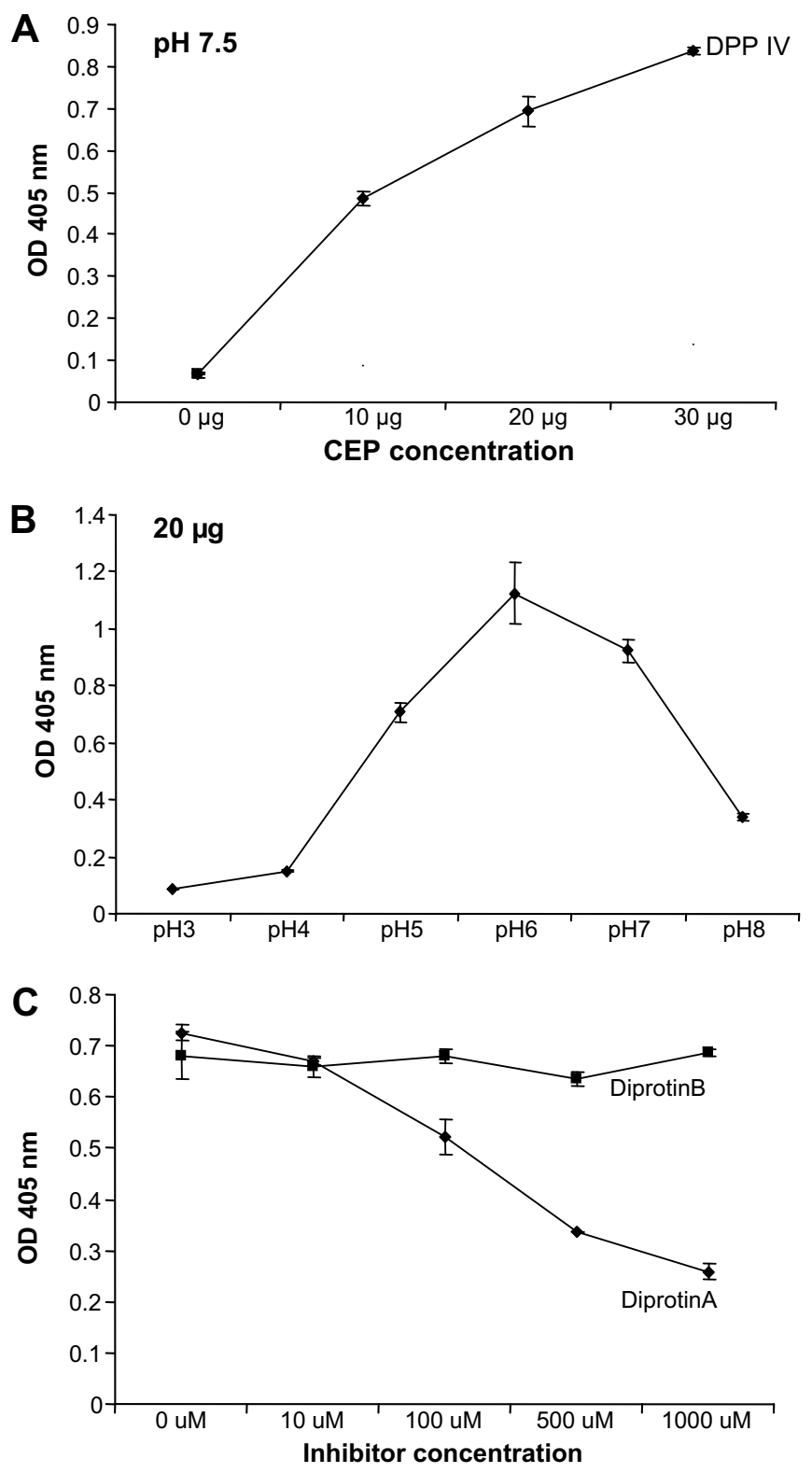

Fig. 4. Peptidase activity assays. (A) Dose-dependent degradation of the dipeptidyl peptidase IV-specific substrate by the CEP at $\mathrm{pH}$ 7.5. (B) Activity against the DPP IV-specific substrate by $20 \mu \mathrm{g}$ of the CEP incubated at different $\mathrm{pH}$. (C) Testing the effect of two DPP IV-specific inhibitors on the DPP IV type activity of the CEP.

tein, which suggested that the $\mathrm{C}$-terminal end of the $\alpha$ chain was degraded. As a control, the $\alpha$-chain of an untreated sample was analysed and this analysis confirmed that $\mathrm{C}$-terminal peptides covering the full $\alpha$-chain sequence could normally be generated.

\subsection{Bt-PEG-Glu-Pro ${ }^{P}(\mathrm{OPh})_{2}$ affinity labelling}

The labelling of CEP with the DPP IV-specific inhibitor Bt-PEG-Glu-Pro ${ }^{\mathrm{P}}(\mathrm{OPh})_{2}$ is shown in Fig. 7A. A strong band is visible around $55 \mathrm{kDa}$, (lane 3), coinciding with the size of the Hc-PCP1 and Hc-PCP2 on a Coomassie- 

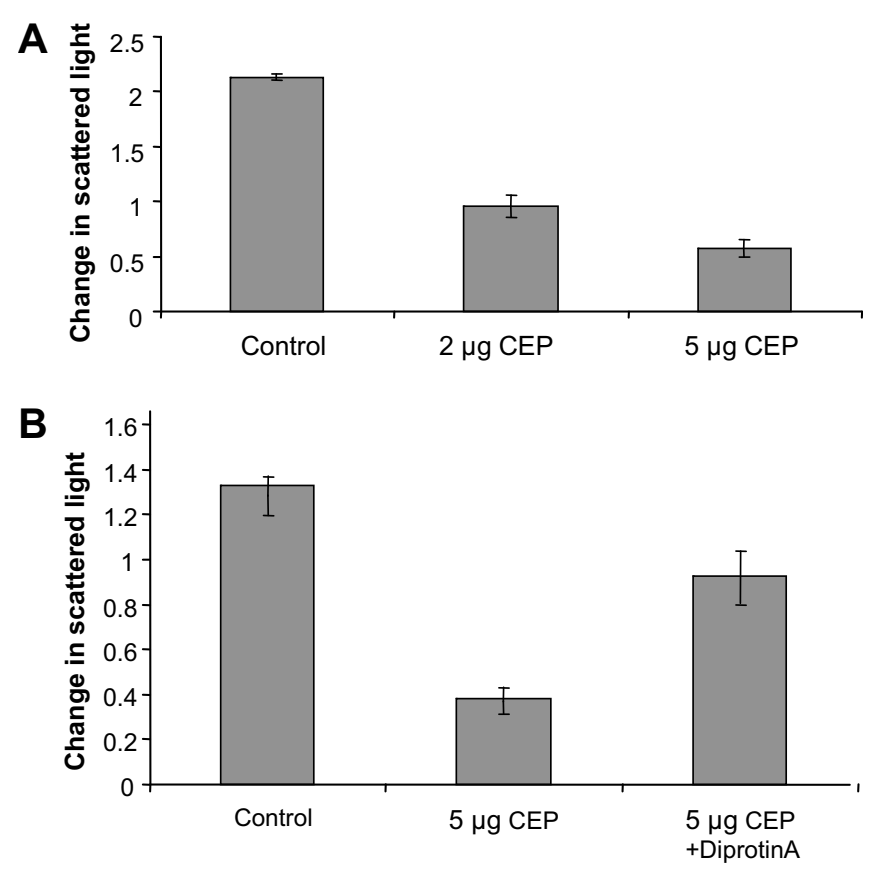

Fig. 5. Inhibition of fibrin re-aggregation by the addition of CEP. (A) The re-aggregation of control fibrin monomers compared with the re-aggregation of fibrin monomers incubated with different concentrations ( 2 and $5 \mu \mathrm{g}$, respectively) of CEP. (B) Analysis of the effect of Diprotin A on the inhibition of fibrin re-aggregation. All results are shown as changes in scattered light measured at $350 \mathrm{~nm}$, which is a measure of the amount of re-aggregated fibrin monomers.

stained gel (lane 1). Other fainter bands were visible, particularly around $38 \mathrm{kDa}$. No bands were recognised in the negative control sample, which consisted of CEP without the addition of Bt-PEG-Glu-Pro ${ }^{\mathrm{P}}(\mathrm{OPh})_{2}$ (lane 2).

\section{Discussion}

The intestinal surface of $H$. contortus is lined by a helical polymeric structure, termed contortin, which fills the spaces between the microvilli (Munn, 1977). In the present study, we have identified the major protein component of the structure as prolyl-carboxypeptidase-like enzymes encoded by two mRNA transcripts, each of which, in turn, encodes two related but distinct PCPs. Consistent with these findings, we show that a protein fraction highly enriched for these enzymes has DPP IV activity, impairs fibrin clot formation and we provide compelling evidence that this property is mediated by DPP IV-dependent cleavage of the alpha-fibrinogen chain. The latter was unaffected by the addition of a cocktail of inhibitors against serine, cysteine, metallo and aspartyl proteases but completely abolished by the addition of DPP IV-specific inhibitors.

The genes (hc-pcp1 and $h c-p c p 2)$ encoding these proteins are both transcribed from the L4 stage onwards. The level of transcript, based on EST levels, is, for Hc-pcpl, extremely high. The Haemonchus EST dataset contains 421 ESTs for Hc-pcpl of which 362 were identified in an intestinal cDNA library. This makes it the third biggest cluster in the total Haemonchus EST dataset (Geldhof et al., 2005) and by far the most abundant EST in the intestinal EST dataset. This is consistent with the observations by Munn (1977) who showed that contortin was a major protein in the intestine of $H$. contortus, present from the L4 stage onwards. The Hc-pcp2 transcript on the other hand seems to be present at a much lower level. Only 37 ESTs are present in the EST dataset of which two were identified in the intestinal cDNA library. This suggests that the contortin structure is likely to be mainly composed of the two PCPs coded by Hc-PCP1. Nevertheless, a peptide specific for PCP2A was also identified in the mass spectrometric analysis of the contortin structure.

The PCPs described in this paper show homology to the peptidase S28 family. This is a family of serine peptidases which include both amino- and carboxy-exopeptidases, examples of which are lysosomal Pro-Xaa carboxypeptidase, dipeptidyl peptidase II and DPP IV. Enzyme activity analyses indicated that DPP IV activity predominated with a $\mathrm{pH}$ optimum at $\mathrm{pH} 6$, activity being substantially inhibited by the competitive DPP IV inhibitor Diprotin A.

Munn (1977) previously proposed that contortin was an immobilised anticoagulant. To investigate this hypothesis we have analysed the anti-clotting activity of CEP against purified fibrin monomers. CEP significantly interfered with fibrin clot formation within seconds after addition. The high rate of this enzymatic reaction might be important for the parasite to immediately prevent blockage of the intestine by the blood meal. The reduced clotting is a result of the degradation of the C-terminal end of the fibrinogen alpha-chain ( $\alpha \mathrm{C}$-domain). Cysteine proteinases have previously been implicated in fibrinogen degradation by extracts of adult $H$. contortus (Boisvenue et al., 1992). Here, addition of specific inhibitors for all four classes of proteinases (serine, cysteine, metallo and aspartyl proteases) to CEP did not inhibit the anti-clotting activity, nor did it have an effect on the carboxypeptidase type degradation of the fibrinogen alpha-chain. Degradation of the alpha-chain was only inhibited when the DPP IV inhibitors Diprotin $\mathrm{A}$ and Bt-PEG-Glu-Pro ${ }^{\mathrm{P}}(\mathrm{OPh})_{2}$ were included in the reaction mixture, results which suggest that the PCPs are responsible for the degradation of the fibrinogen alphachain. However, apart from the PCPs, the use of the biotinylated DPP IV inhibitor also resulted in the labelling of some fainter bands around $38 \mathrm{kDa}$. At this stage it is unclear if this is background recognition or if it is actually caused by the presence of additional DPP IV like enzymes in the CEP fraction. If this is the case, we cannot exclude their involvement in the fibrinolytic activity.

Blood-feeding parasites have developed an array of specific inhibitors to interfere with mammalian blood coagulation (Ledizet et al., 2005). Most of these inhibitors target the serine proteases involved in the early steps of the clotting cascade. To date, a number of anticoagulants have been identified in both hookworms and ticks which inhibit the coagulation factor $\mathrm{Xa}$, the prothrombinase complex and the fVIIa/tissue factor complex (Mans and Neitz, 


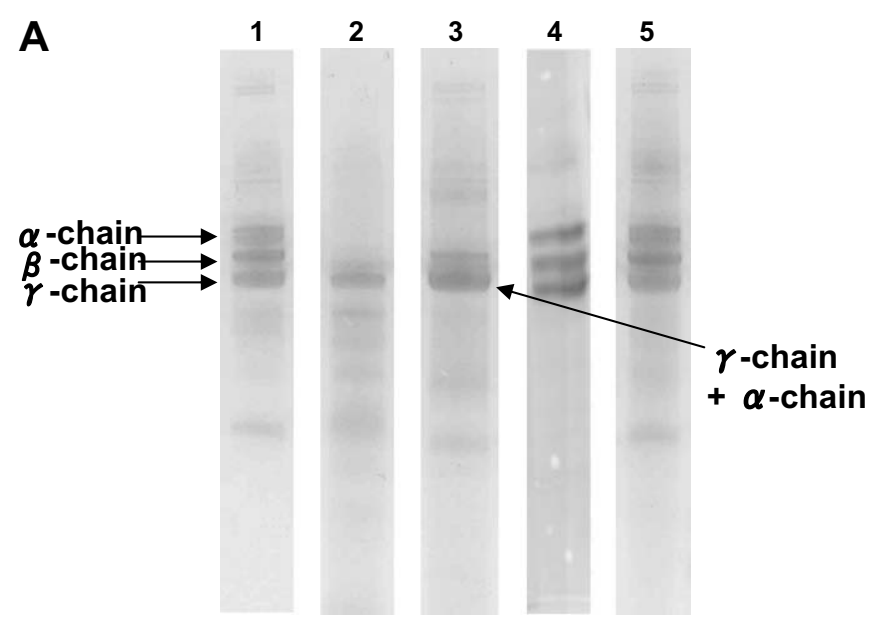

B

MFSVRDLCLVLSLVGAIKTEDGSDPPSGDFLTEGGGVRGPRLVERQQSACKETGWPFCS DEDWNTKCPSGCRMKGLIDEVDQDFTSRINKLRDSLFNYQKNSKDSNTLTKNIVELMRG DFAKANNNDNTFKQISEDLRSRIEILRRKVIEQVQRIKVLQKNVRDQLVDMKRLEVDIDIKIR SCKGSCSRALEHKVDLEDYKNQQKQLEQVIAINLLPSRDIQYLPLIKMSTITGPVPREFKSQ LQEAPLEWKALLEMQQTKMVLETFGGDGHARGDSVSQGTGLAPGSPRKPGTSSIGNVNP GSYGPGSSGTWNPGRPEPGSAGTWNPGRPEPGSAGTWNPGRPEPGSAGTWNPGRPE PGSAGTWNPGRPEPGSAGTWNTGSSGSSSFRPDSSGHGNIRPSSPDWGTFREEGSVSS GTKQEFHTGKLVTTKGDKELLIDNEKVTSGHTTTTRRSCSKVITKTVTNADGRTETTKEVV KSEDGSDCGDADFDWHHTFPSRGNLDDFFHRDKDDFFTRSSHEFDGRTGLAPEFAALGE SGSSSSKTSTHSKQFVSSSTTVNRGGSAIESKHFKMEDEAESLEDLGFKGAHGTQKGHTK ARPARGIHTSPLGEPSLTP

\section{Control}

\section{TVTNADGRTETTKEVVKSEDGSDCGDADFDWHHTFPSRGNLDDFFHRDKD DFFTRSSHEFDGRTGLAPEFAALGESGSSSSKTSTHSKQFVSSSTTVNRGGSAIESKHFK MEDEAESLEDLGFKGAHGTQKGHTKARPA RGIHTSPLGE PSLTP}

Fig. 6. Proteolytic degradation of the fibrin $\alpha$-chain by CEP and the inhibition of this activity by dipeptidyl IV-specific inhibitors Diprotin A and Bt-PEGGlu-Pro ${ }^{\mathrm{P}}(\mathrm{OPh})_{2}$. (A) $10 \%$ reducing SDS-PAGE gel of a control fibrin solution $(10 \mu \mathrm{g})$ (lane 1), fibrin $(10 \mu \mathrm{g})$ incubated with CEP for $15 \mathrm{~s}(\mathrm{lane} 2)$, fibrin $(10 \mu \mathrm{g})$ incubated for $15 \mathrm{~s}$ with CEP in the presence of E64, 1,10 Phe, AEBSF and Pepstatin (lane 3), fibrin (10 $\mu \mathrm{g})$ incubated for $15 \mathrm{~s}$ with CEP preincubated with the DPP IV-specific inhibitors Diprotin A (lane 4) and Bt-PEG-Glu-Pro ${ }^{\mathrm{P}}(\mathrm{OPh})_{2}$ (lane 5). (B) Complete peptide sequence of the bovine fibrinogen $\alpha$-chain (P02672). The peptides highlighted were identified by mass-spectrometry analysis or LC/MS-MS in the fibrinogen $\gamma$-band after incubation with CEP. The peptides identified in the C-terminal end of a control fibrinogen $\gamma$-band (without incubation with the PCP-enriched protein fraction) are highlighted separately. The results suggest that the C-terminal end of the $\alpha$-chain was degraded by the PCP activity.

2004; Mieszczanek et al., 2004). The anticoagulant activity in CEP, most likely due to the PCPs, targets the key structural component of the blood clot itself, fibrinogen. The structure of fibrinogen and fibrin has recently been reviewed by Mosesson (2005). In short, fibrinogen has a dimeric structure of with each monomer composed of $\alpha-$, $\beta$ - and $\gamma$-chain. The $\mathrm{N}$-terminal domains of the six chains in a fibrinogen molecule are linked together by disulphide bonds to form the E-domain. When clotting occurs, thrombin cleaves off the fibrinopeptides at the $\mathrm{N}$-terminal ends of the $\alpha$ - and $\beta$-chain. The newly exposed $\mathrm{N}$-terminal ends of these chains will respectively bind to the terminal $\gamma \mathrm{C}$-and $\beta \mathrm{C}$-domain of an adjacent fibrinogen molecule and form fibrin. Multiple fibrin molecules will align to form fibrils and these will undergo lateral associations with other fibrils to create multi-stranded fibres and eventually the clot. Here, degradation specifically targets the $\alpha \mathrm{C}$-domain. As far as we know, it is the first time this type of anti-thrombotic activity has been identified in any organism. Some serine and metallo-proteases have been identified in snake venom with a substrate preference for the fibrinogen $\alpha$ chain (Matsui et al., 2000) and a dipeptidyl peptidase IV has been identified in human placenta which cleaved the glycylproline residues from the $\mathrm{N}$-terminal end of the fibrin $\alpha$-chain (Mentlein and Heymann, 1982). However, the specificity for the $\alpha \mathrm{C}$-domain seems to be unique. This part of the $\alpha$-domain has shown to be important for both intraand intermolecular interactions in fibrin and fibrils (Mosesson, 2005). Fibrinogen molecules lacking this domain display a slower rate of assembly, a reduced turbidity and generate thinner fibres (Mosesson, 2005). The $\alpha \mathrm{C}$-domains are normally non-covalently associated with the central Edomain. When thrombin cleaves the fibrinopeptide B's, the aC-domains dissociates from this E-domain and become 


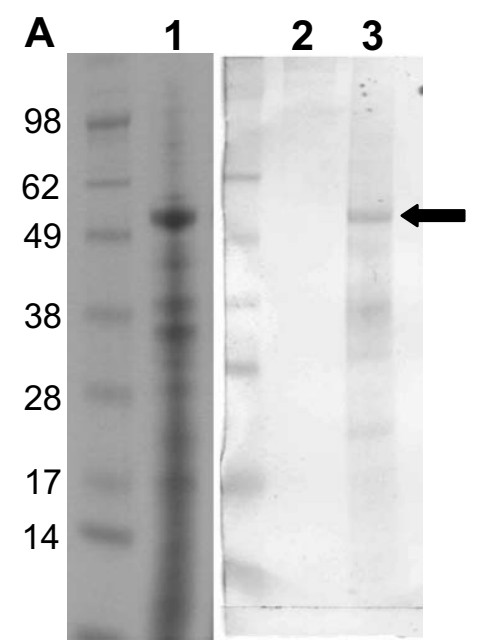

Fig. 7. Affinity labelling of contortin-enriched preparation (CEP) with the dipeptidyl peptidase IV (DPP IV)-specific inhibitor Bt-PEG-Glu-Pro$\mathrm{P}(\mathrm{OPh})_{2}$. Coomassie and Western blot analysis of CEP. Lane 1: $10 \mu \mathrm{g}$ CEP on Coomassie-stained SDS-PAGE. Lanes 2 and 3: $10 \mu \mathrm{g}$ CEP on Western blot developed with streptavidin conjugate, respectively, with and without the addition of Bt-PEG-Glu-. The PCP band is marked with an arrow.

available for interaction with other $\alpha \mathrm{C}$-domains (Mosesson, 2005). This increased sterical accessibility of the $\alpha \mathrm{C}$ domain might be the reason why it is targeted by the PCPs.

The high intestinal transcription levels of the PCPs seem to be very specific for $H$. contortus. Using the Haemonchus PCP sequences as search queries, we identified EST clusters from A. caninum, A. suum, $O$. volvulus, $S$. ratti and $P$. trichosuri that encode for putative PCPs. However, each of these clusters contained a small number of ESTs, ranging from one to six ESTs, indicating an overall low transcription level. In addition, no PCPs have been identified in the intestinal EST libraries from the two hookworm species Necator americanus and A. caninum (Ranjit et al., 2006) and no new sequence homologues were identified in a recent search of the databases. Munn (1977) previously suggested, based on microscopic observations, the presence of a contortin-like structure in the intestine of the sheep parasite Teladorsagia circumcincta. However, analysis of a $T$. circumcincta protein extract, purified according to the methods described by Munn (1977), showed a totally different protein profile and did not contain any detectable levels of DPP IV activity (P. Geldhof and D. Knox, unpublished data).

The data here indicate that PCPs potentially play a crucial role in the survival of $H$. contortus. Despite a successful vaccination trial with the CEP fraction (Munn et al., 1987), it is unclear what the protective capacity is of the PCPs themselves. The analysis of CEP here showed the presence of several other protective antigens, such as $\mathrm{H} 11$, an apical gut membrane protein, cysteine proteinases and metalloprotease III, all of which could have been implicated in vaccine-induced protection against Haemonchus challenge. Further purification is required to fully analyse the protective capacity of the PCPs.
Given their apparently novel mode of action, PCPs might have a potential as drug targets. In recent years, much attention has been given in the medical research field to the development of inhibitors for this type of enzyme, in particular for DPP IV (Rosenblum and Kozarich, 2003). Such inhibitors, when available, could easily be tested in the coagulation assays for their inhibitory activity of the $H$. contortus PCPs. Moreover, the $H$. contortus PCPs might also have medical applications. A number of anti-thrombotics from haematophagous invertebrates have been evaluated in vivo for the treatment of a variety of conditions associated with blood clotting. Some of these are already in varying stages of preclinical and clinical development, for example the nematode anticoagulant protein-2 (Ledizet et al., 2005).

\section{Acknowledgements}

P.G. is a Postdoctoral Fellow of the Fund for Scientific Research - Flanders (Belgium) (F.W.O. - Vlaanderen). We thank Dr. Brendan Gilmore for the biotinylated protease inhibitor.

\section{References}

Boisvenue, R.J., Stiff, M.I., Tonkinson, L.V., Cox, G.N., Hageman, R., 1992. Fibrinogen-degrading proteins from Haemonchus contortus used to vaccinate sheep. Am. J. Vet. Res. 53, 1263-1265.

Geldhof, P., Whitton, C., Gregory, W.F., Blaxter, M., Knox, D.P., 2005. Characterisation of the two most abundant genes in the Haemonchus contortus expressed sequence tag dataset. Int. J. Parasitol. 35, 513-522.

Gilmore, B.F., Carson, L., McShane, L.L., Quinn, D., Coulter, W.A., Walker, B., 2006. Synthesis, kinetic evaluation, and utilization of a biotinylated dipeptide proline diphenyl phosphonate for the disclosure of dipeptidyl peptidase IV-like serine proteases. Biochem. Biophys. Res. Commun. 347, 373-379.

Knox, D.P., Redmond, D.L., Newlands, G.F., Skuce, P.J., Pettit, D., Smith, W.D., 2003. The nature and prospects for gut membrane proteins as vaccine candidates for Haemonchus contortus and other ruminant trichostrongyloids. Int. J. Parasitol. 33, 1129-1137.

Ledizet, M., Harrison, L.M., Koskia, R.A., Cappello, M., 2005. Discovery and pre-clinical development of antithrombotics from hematophagous invertebrates. Curr. Med. Chem. Cardiovasc. Hematol. Agents 3, 110 .

Liddell, S., Knox, D.P., 1998. Extracellular and cytoplasmic $\mathrm{Cu} / \mathrm{Zn}$ superoxide dismutases from Haemonchus contortus. Parasitology 116, 383-394

Mans, B.J., Neitz, A.W., 2004. Adaptation of ticks to a blood-feeding environment: evolution from a functional perspective. Insect Biochem. Mol. Biol. 34, 1-17.

Matsui, T., Fujimura, Y., Titani, K., 2000. Snake venom proteases affecting hemostasis and thrombosis. Biochim. Biophys. Acta 1477, $146-156$.

Mentlein, R., Heymann, E., 1982. Dipeptidyl peptidase IV inhibits the polymerization of fibrin monomers. Arch. Biochem. Biophys. 217, $748-750$.

Mieszczanek, J., Harrison, L.M., Cappello, M., 2004. Ancylostoma ceylanicum anticoagulant peptide-1: role of the predicted reactive site amino acid in mediating inhibition of coagulation factors Xa and VIIa. Mol. Biochem. Parasitol. 137, 151-159.

Mosesson, M.W., 2005. Fibrinogen and fibrin structure and functions. J. Thromb. Haemost. 3, 1894-1904. 
Munn, E.A., 1977. A helical, polymeric extracellular protein associated with the luminal surface of Haemonchus contortus intestinal cells. Tissue Cell 9, 23-34.

Munn, E.A., Greenwood, C.A., Coadwell, W.J., 1987. Vaccination of young lambs by means of a protein fraction extracted from adult Haemonchus contortus. Parasitology 94, 385-397.

Parkinson, J., Anthony, A., Wasmuth, J., Schmid, R., Hedley, A., Blaxter, M., 2004. artiGene - constructing partial genomesBioinformatics 20, 1398-1404.

Ranjit, N., Jones, M.K., Stenzel, D.J., Gasser, R.B., Loukas, A., 2006. A survey of the intestinal transcriptomes of the hookworms, Necator americanus and Ancylostoma caninum, using tissues isolated by laser microdissection microscopy. Int. J. Parasitol. 36, 701-710.
Rosenblum, J.S., Kozarich, J.W., 2003. Prolyl peptidases: a serine protease subfamily with high potential for drug discovery. Curr. Opin. Chem. Biol. 7, 496-504.

Smith, T.S., Munn, E.A., 1990. Strategies for vaccination against gastrointestinal nematodes. Rev. Sci. Tech. 9, 577-595.

Underwood, R., Chiravuri, M., Lee, H., Schmitz, T., Kabcenell, A.K., Yardley, K., Huber, B.T., 1999. Sequence, purification, and cloning of an intracellular serine protease, quiescent cell proline dipeptidase. $\mathbf{J}$. Biol. Chem. 274, 34053-34058.

Willadsen, P., Bird, P., Cobon, G.S., Hungerford, J., 1995. Commercialisation of a recombinant vaccine against Boophilus microplus. Parasitology 110, S43-S50. 